\title{
Frühzeitig Eisenchelat-Therapie bei Transfusionsabhängigkeit einleiten
}

\begin{abstract}
Hämoglobin-Anomalien wie Thalassämie oder die Sichelzellanämie erfordern lebenslange Transfusionen meist bereits ab dem Kindesalter. Hierdurch kommt es zu einer Eisenüberladung, die eine begleitende Eisenchelat-Therapie erforderlich macht.
\end{abstract}

Unter den rund 16 Mio. Einwohnern mit Migrationshintergrund in Deutschland sind etwa 4,8\% Anlagenträger einer Hämoglobin-Anomalie, wie der Thalassaemia major oder der Sichelzellenanämie. Kinder mit angeborener $\beta$-Thalassämie sind früher nur etwa fünf Jahre alt geworden. Eine kurative Therapie ist heute mit einer Stammzelltransplantation möglich. Ziel der Transfusionsbehandlung ist, so Holger Cario vom Universitätsklinikum Ulm, die Kompensation der Anämie mit Suppression der Eigenerythropoese.
Mit der Transfusion eines Erythrozytenkonzentrats werden jedoch etwa $250 \mathrm{mg}$ Eisen zugeführt. Weil der Mensch überschüssiges Eisen nicht eliminieren kann, kommt es bereits ab der Zufuhr von 10 bis 15 Einheiten Erythrozytenkonzentrat zu einer relevanten Eisenüberladung. Die Folgen sind vielfältig, sie betreffen beispielsweise Herz, Leber, Pankreas, Schilddrüse, Hypophyse, Gonaden, Nebennierenrinde und Knochen.

In der Wachstumsphase reagiert der Organismus offenbar besonders empfindlich auf eine Eisenüberladung, sodass es nach Aussage von Regine Grosse vom Universitätsklinikum HamburgEppendorf, wichtig ist, schon im Kindesalter mit einer Eisenchelator-Therapie zu beginnen. Hiermit gelang es in den letzten Jahren, das Überleben der Patienten mit Thalassaemia major deutlich zu verlängern.
Zur Therapie stehen das subkutan oder intravenös applizierbare Deferoxamin $\left(\right.$ Desferal $\left.^{\circledR}\right)$ sowie die beiden oralen Eisen-Chelatoren Deferipron (Ferriprox ${ }^{\circledast}$ ) und Deferasirox (Exjade ${ }^{\circledast}$ zur Verfügung. Deferipron muss dreimal täglich eingenommen werden. Das Risiko einer Neutropenie bzw. Agranulozytose erfordert regelmäßige Blutbildkontrollen. Deferasirox muss nur einmal täglich eingenommen werden.

Die Substanz steht auch in Form einer anwenderfreundlichen Brausetablette zur Verfügung. Als unerwünschte Wirkung kann es zum Anstieg des Kreatininspiegels kommen. „Die Eisenchelator-Therapie ist ein Segen für die Patienten, sie ist aber kompliziert und muss überwacht werden“, so Grosse zusammenfassend.

SUH

"Meet the Experts" bei der DGKJ-Jahrestagung am 23. September 2011 in Bielefeld; Veranstalter: Novartis Pharma

\section{Fortgeschrittenes nicht plattenepitheliales NSCLC Positives Votum für Pemetrexed als Erhaltungstherapie}

\section{Das CHMP (Committee for Mecical Products for Human Use) der Europäischen Arzneimittelagentur (EMA) empfiehlt die Zulassung von Pemetrexed (Alimta ${ }^{\circledR}$ ) zur Erhaltungstherapie von Patienten mit fortgeschrittenem nicht plattenepithelialem NSCLC.}

Basis der Empfehlung sind die Daten der PARAMOUNT-Studie [J Clin Oncol 2011; 29 (Suppl): Abstract CRA 7510]. In dieser Phase-III-Studie wurden 939 Patienten mit fortgeschrittenem nicht plattenepithelialem NSCLC in der First-Line zunächst mit dem Standard Pemetrexed/Cisplatin behandelt. Im Fall einer kompletten oder partiellen Tumorrückbildung bzw. einer Krankheitsstabilisierung erfolgte dann eine Erhaltungstherapie: randomisiert wurde die Pemetrexed-Therapie fortgeführt oder die Patienten bekamen Placebo.

Alle Patienten wurden palliativ versorgt und erhielten zusätzlich Vitamin B12, Folsäure sowie Dexamethason. Primärer Studienendpunkt war das progressionsfreie Überleben (PFS) ab Beginn der Erhaltungstherapie.
Die wichtigsten Ergebnisse:

- In der Pemetrexed-Gruppe betrug das PFS im Mittel 4,1 Monate, unter Placebo 2,8 Monate; der Unterschied war signifikant ( $\mathrm{p}=0,00006)$, HR 0,62).

- Das Progressionsrisiko war unter Pemetrexed um $38 \%$ niedriger (vs. Placebo HR 0,62).

Für Studienleiter Luis Paz-Ares zeigt die PARAMOUNT-Studie, dass Patienten nach erfolgreicher Erstlinientherapie mit Pemetrexed/Cisplatin von der Weiterbehandlung mit Pemetrexed profitieren können.

Nach Informationen der Lilly Deutschland 